\title{
Comprensión de oraciones de esfuerzo en jóvenes y adultos mayores desde una perspectiva corpórea*
}

\section{Comprehension of Effort's Sentences in Young and Older Adults from an Embodiment Perspective}

Recibido: 03 Enero 2018 | Aceptado: 30 Noviembre 2020

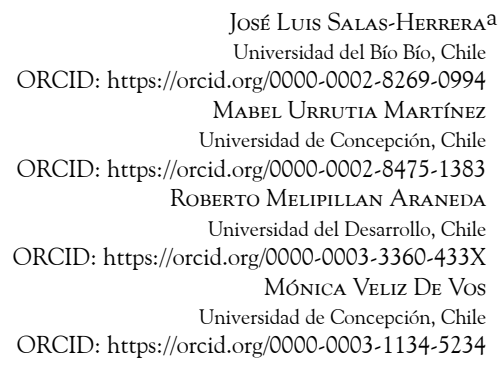

a Autor de correspondencia. Correo electrónico: jsalas@ubiobio.cl

Para citar este artículo: Salas-Herrera, J. L., Urrutia, M., Melipillan, R., Veliz, M. (2020). Comprensión de oraciones de esfuerzo en jóvenes y adultos mayores desde una perspectiva corpórea. Universitas Psychologica, 19. https://doi.org/10.11144/Javeriana.u psy19.coej

\section{RESUMEN}

Actualmente se desconoce la validez de las propuestas de la cognición corpórea y su relación con el lenguaje de los adultos mayores. Para responder a esto realizamos un experimento con los factores Esfuerzo, Imaginabilidad y Contexto Lingüístico. 50 adultos mayores $(M=66.18$ años, $\mathrm{DE}=4.39,22$ mujeres y 28 hombres $)$ y 43 jóvenes $(M=21.28$ años, $\mathrm{DE}=1.08,36$ mujeres y 7 hombres) leyeron oraciones en la pantalla de un computador, presionando la barra espaciadora para decidir luego si una palabra se hallaba o no en la frase recién leída. Los resultados muestran efectos principales para los factores Imaginabilidad $(\beta=0.309$; $p<0.05)$ y Contexto $(\beta=-0.856 ; p<0.001)$ en los tiempos de lectura del objeto directo, dos efectos de interacción Imaginabilidad-Esfuerzo $(\beta=$ -0.732; $p<0.01)$ e Imaginabilidad-Contexto $(\beta=0.611 ; p<0.05)$ para el complemento circunstancial y un efecto interactivo ImaginabilidadContexto $(\beta=0.727 ; p<0.05)$ para la palabra de activación. Los resultados respaldan una visión de corporeidad débil con integración interactiva de las propiedades corpóreas y simbólicas de los textos. Es necesario ampliar la investigación a otros parámetros corpóreos, edades y lenguas para contrastar estos resultados.

Palabras clave

corporeidad; envejecimiento; esfuerzo; imaginabilidad; contrafactuales.

\begin{abstract}
Currently, the validity of the proposals of embodiment cognition and its relationship with the language of older adults is unknown. To answer this, we conducted an experiment with the Effort, Imaginability, and Linguistic Context factors. 50 older adults $(M=66.18$ years, $S D=4.39,22$ women and 28 men) and 43 young people $(M=21.28$ years, $S D=1.08,36$ women and $7 \mathrm{men}$ ) read sentences on a computer screen, pressing the space bar to then decide whether or not a word was in the sentence just read. The results show main effects for the Imaginability $(\beta=0.309 ; p$ $<0.05)$ and Context factors $(\beta=-0.856 ; \mathrm{p}<0.001)$ in the reading times
\end{abstract}


of the direct object, two effects of Imaginability-Effort interaction $(\beta=-0.732 ; p<0.01)$ and ImaginabilityContext $(\beta=0.611 ; p<0.05)$ for the circumstantial complement, and one interactive Imaginability-Context effect $(\beta=0.727 ; p<0.05)$ for the activation word. The results support a vision of weak corporeality with interactive integration of the corporeal and symbolic properties of the texts. It is necessary to extend the research to other embodiment parameters, ages, and languages to contrast these results.

Keywords

embodiment; aging; effort; imaginability; counterfactual.

\section{Envejecimiento, lenguaje y corporeidad}

La cognición corpórea ha adquirido mucho prestigio en las últimas décadas y, en el plano del lenguaje, plantea que la comprensión y producción de este están basados en los sistemas de acción, percepción y emoción (Johnson, 2018; Ostarek \& Huettig, 2019). La comprensión del discurso estaría influida por diversos procesos y parámetros de la experiencia humana (Zwaan, 2014). La agenda investigativa se ha centrado, principalmente, en el nivel de participación del cuerpo en la cognición y el lenguaje (Tirado et al., 2018), el rol causal de áreas cerebrales extraperisilvánicas en la comprensión del lenguaje, por ejemplo, la corteza motora primaria (Vukovic et al., 2017), sensorial y parietal (Yang et al., 2017) y el problema de la corporeización de los conceptos abstractos (Borghi et al., 2019; Borghi et al., 2017).

Se ha demostrado que ensayar mentalmente un ejercicio físico se relaciona con un nivel de esfuerzo que induce un aumento de la fuerza muscular comparable a la alcanzada por un ejercicio real (Di Rienzo et al., 2019; Goudarzian et al., 2017). Entre los parámetros corpóreos que influyen en la percepción, acciones, simulación mental y, consecuentemente, en la comprensión del discurso estarían la dirección, perspectiva, distancia, posición, región espacial, dolor y, justamente, el nivel de esfuerzo implicado en las acciones humanas (Zwaan, 2014) interactuando con un entorno sobre la base de las disponibilidades o affordances que se generan (Gibson, 1979). Estudiar todos estos parámetros sería complejo por lo que se optó por investigar uno que se encuentra relativamente poco explorado en la literatura corpórea: el parámetro del esfuerzo. El nivel de esfuerzo es un parámetro que aplica a cada tarea motora y mental que se ejecute, y los grados de fuerza necesarios para llevar a cabo la acción física o mental son sus valores (Gallese \& Lakoff, 2005).

Ahora bien, desde un punto de vista lingüístico, algunas de las teorías corpóreas que permiten comprender cómo se vinculan los parámetros corpóreos con la comprensión del discurso está la Hipótesis Indexical (Glenberg \& Robertson, 2000; Mohan et al., 2016) y la Teoría de Lenguaje Basado en la Acción (LBA) (Glenberg \& Gallese, 2012; Sadoski, 2018). La Hipótesis Indexical propone que el significado que una situación tiene para un individuo se vincula con el conjunto de acciones potenciales (affordances) que ese individuo puede realizar en dicha situación (Gibson, 1979). Las palabras son indexadas (mapeadas) a los referentes presentes en el entorno o al sistema de símbolos perceptuales (Barsalou, 1999) que se ha generado y mantenido para aquellos objetos en el mundo real. La experiencia consciente permite generar estos símbolos que, al mismo tiempo que guardan relación con los referentes originales, participan de procesos como la memoria, el pensamiento y el lenguaje a través de recombinaciones que permiten construir "simulaciones" de la experiencia. La organización interna de estos símbolos es modal y ellos están analógicamente relacionados con los objetos, referentes, estados o situaciones que permitieron su generación (Louwerse, 2018).

Por otro lado, Glenberg y Gallese (2012) proponen la Teoría LBA, que intenta explicar la adquisición, comprensión y producción del lenguaje (principalmente gestos), adaptando las teorías del control motor MOSAIC (selección modular e identificación para el control) y HMOSAIC (selección modular jerárquica e identificación para el control) propuestas por Wolpert et al. (2003). Los autores relacionan algunas de las operaciones motoras con las neuronas espejo y canónicas, para luego aplicar estas operaciones al procesamiento del lenguaje. En concreto, el modelo plantea 
la existencia de controladores, predictores, procesos comparadores y de retroalimentación sensorial. Los controladores comandan los efectores -incluyendo los del habla- en un entorno físico y social buscando responder a las demandas mediante un ajuste pragmático. Una copia de los comandos usados, en términos de configuraciones, es enviada directamente a los predictores, los que cumplen la función de anticipar tanto los movimientos de los otros como de la propia persona, proveyendo un modelo de lo esperado que permite solventar la demora de la retroalimentación sensorial. Las señales del controlador y la retroalimentación sensorial son ponderadas por un comparador cuya función es enviar señales de ajuste/error que permitan mantener/modificar la acción en curso (vía controladores). Las simulaciones están guiadas por los modelos de acción internos articulados por los predictores, basados a su vez en el sistema motor, sensorial y emocional (Glenberg et al., 2013). En esta teoría, la predicción basada en la simulación motora y anclada en la experiencia individual tiene un peso importante en la comprensión del lenguaje.

\section{El problema de la abstracción}

El plano de las simulaciones y modelos mentales relacionados con los discursos abstractos, ligados a acciones cognitivas o mentales, que por definición son menos corpóreas (Borghi et al., 2019; Borghi et al., 2017), es uno de uno de los retos a los que se enfrenta la visión de la simulación experiencial de la comprensión del lenguaje. Al respecto, varios autores (p.e. Ostarek \& Huettig, 2019) han propuesto que la información abstracta se mapea en dominios experienciados para obtener significado. Recientemente, se ha sugerido que la comprensión de palabras abstractas tiene su base en la reactivación de experiencias afectivas (Ponari et al., 2018). Otro dominio en el que los conceptos abstractos estarían mapeados es el espacial (Jamrozik et al. 2016; Marmolejo-Ramos et al., 2017).
Tomando esta evidencia en conjunto, se puede aseverar que la información abstracta puede ser capturada en representaciones experienciales por medio de asignaciones metafóricas. En todo caso, sostener que dichas asignaciones son funcionalmente relevantes para la comprensión del lenguaje es complejo y requiere mayor indagación (Ulrich et al., 2012). Además, se encuentra el argumento desarrollado por Paivio (1986), quien señala que las palabras abstractas se procesan en el sistema verbalsimbólico y las concretas en el sistema de imágenes mentales, admitiendo así un sistema de representación simbólica y otro corpóreo, para procesar ambos tipos de palabras en una suerte de sistema híbrido (Ostarek et al., 2019). En este sentido, es importante mencionar que el contexto lingüístico-comunicativo es muy relevante al momento de entender el lenguaje abstracto en el continuo que va desde el polo corpóreo al descorporeizado (Borghi et al., 2019; Ströbel, 2016).

\section{Lenguaje contrafactual y corporeidad}

Una construcción lingüística abstracta que ha venido estudiándose progresivamente más por los investigadores corpóreos es la del lenguaje contrafactual, que forma parte de los predicados con significados mentalistas (Kulakova \& Nieuwland, 2016). El lenguaje contrafactual es omnipresente en la vida cotidiana (Roese \& Epstude, 2017) y tiene varias funciones adaptativas. Por ejemplo, permite razonar sobre las causas de un evento (Nyhout et al., 2019), desempeñando así un rol importante en el aprendizaje de la experiencia, ya que promueve emociones como el arrepentimiento y el alivio, colaborando en la regulación del comportamiento y las emociones para poder funcionar adecuadamente en los entornos físico y social (Roese \& Epstude, 2017). Además, este está asociado con la comprensión de las perspectivas y creencias de los demás, lo cual podría calificarlo como precursor del desarrollo de los procesos implicado en la teoría de la mente (Guajardo \& Cartwright, 2016). El lenguaje 
contrafactual es, por ende, evidencia de una función cognitiva altamente compleja que se desarrolla relativamente tarde en la infancia (Nyhout et al., 2019).

La razón por la cual los contrafactuales son cognitivamente complejos es porque generan dos representaciones incompatibles (Romoli et al., en prensa). Por ejemplo, "si tuviera branquias, entonces podría respirar bajo el agua”, expresa (1) el estado suposicional pero factualmente falso del hablante que tiene branquias y que puede respirar bajo el agua, mientras que también expresa (2) que el hablante no tiene branquias y, por lo tanto, se basa en el modo convencional de respiración humana. Este doble significado es el rasgo cardinal de los contrafactuales. Desde un punto de vista lingüístico, este doble sentido hace de la contrafactualidad un fenómeno fascinante que permite a las personas producir enunciados que son factualmente falsos y sinceros a la vez. Los contrafactuales, por lo tanto, amplían el alcance de la comunicación y permiten conversaciones significativas sobre temas que van más allá de simples declaraciones verídicas (Kulakova \& Nieuwland, 2016).

En años recientes, el lenguaje contrafactual ha sido estudiado desde la perspectiva corpórea (Salas-Herrera, 2015). Indagando en los mecanismos cerebrales y la naturaleza de las representaciones semánticas involucradas en la comprensión del significado complejo de declaraciones contrafactuales, que describen acciones de alto y bajo esfuerzo físico, Urrutia et al. (2012) compararon las respuestas a dichos enunciados con declaraciones fácticas similares usando fMRI. Entre sus hallazgos está que las oraciones de alto esfuerzo físico activan zonas cerebrales implicadas en la comprensión y planificación de acciones, distinta de las redes activadas por las declaraciones factuales. A la fecha, no obstante, no se dispone de estudios sobre declaraciones contrafactuales mentales que correspondan a un nivel de abstracción más alto y que, por tanto, trasciendan el lenguaje de acción, profusamente estudiado por el enfoque corpóreo.

\section{El esfuerzo como un parámetro neural y corpóreo}

En relación con el parámetro neuronal y corpóreo del esfuerzo, investigaciones anteriores han demostrado que la aplicación de diferentes grados de fuerza en el desempeño de la acción provoca actividad diferencial en regiones sensoriomotoras (p.e. Kenville et al., 2017). Los resultados de los estudios citados sugieren que el lenguaje puede evocar representaciones asociadas con el peso de los objetos (Scorolli et al., 2009). En el estudio, los participantes escucharon oraciones referentes al levantamiento de objetos livianos o pesados (p.e. almohada o cofre, respectivamente); posteriormente, levantaron una de dos cajas que eran visualmente idénticas, aunque una era liviana y la otra pesada. Los resultados indicaron que los participantes fueron más lentos cuando el peso sugerido por la frase y el peso de la caja correspondían, este efecto se interpretó como evidencia de que el lenguaje puede activar una simulación sensoriomotora sensible a propiedades intrínsecas tales como el peso de los objetos. Por otro lado, desde el punto de vista del desarrollo humano, son escasos los estudios que han investigado la corporeidad del lenguaje en personas mayores. Entre dichos estudios se encuentra el realizado por de Scalzi et al (2015), que verificó que en adultos mayores sanos se encuentra preservado el efecto de compatibilidad acción-oración (ACE), evidenciando que la comprensión de una oración puede facilitar o interferir con una acción física. La ocurrencia de este efecto en personas mayores evidenciaría que los efectos de la cognición corpórea en el lenguaje están guiados por una reactivación de asociaciones implícitas previamente aprendidas o construidas (Loeffler et al., 2016). Por su parte, Dijkstra et al (2004) pidieron a participantes mayores y jóvenes leer oraciones sobre objetos y luego se les mostró la imagen de un objeto que coincidía y que no coincidía con la forma implícita del objeto en la oración. Las respuestas fueron más rápidas en la condición de ajuste entre formas para todos los participantes, pero el efecto de desajuste fue más fuerte para los adultos mayores. A 
partir de estos datos los autores interpretaron que los adultos mayores construirían modelos de situación más fuertes que los jóvenes. También podría interpretarse que se produce algún nivel de desajuste que se intensifica con la edad cuando se presentan alteraciones o desbalances en la relación lenguaje, cuerpo y contexto, idea que sustenta la presenta investigación.

Igualmente, es posible elaborar algunas hipótesis inspiradas en otras funciones cognitivas relevantes para el parámetro del esfuerzo tales como la predicción de acciones corporales y la estimación en el levantamiento de peso. Diersch et al (2012) encontraron que, en el curso del tiempo, los adultos mayores predicen con menos precisión ciertas acciones de la vida diaria, pero que dicha imprecisión se atenúa en acciones donde las personas mayores hayan acumulado un alto nivel de experiencia sensoriomotriz. Igualmente se ha visto que el procesamiento del lenguaje, vía simulación sensoriomotora, afecta las predicciones de las acciones de otros (Springer et al., 2012). En virtud de la relación bidireccional entre acción y lenguaje y de su sustrato neuronal común, es posible afirmar que la predicción de acciones desencadenará simulaciones de acción que afectarán el procesamiento lingüístico.

En relación con el levantamiento de objetos pesados, tarea donde está implicado el parámetro del esfuerzo, se ha comprobado que el peso es una propiedad intrínseca que también participa de las simulaciones sensoriomotoras del lenguaje (Scorolli et al., 2009). En una investigación en la que se pidió a jóvenes y mayores estimar el peso de cajas que eran levantadas, Maguinness et al (2013) descubrieron que los participantes mayores fueron menos sensibles al discriminar el peso de las cajas levantadas, un efecto que fue paradójicamente más pronunciado en la condición de cajas livianas. El rendimiento en la discriminación de peso fue mejor para las cajas pesadas en comparación con las livianas en ambos grupos, lo que se explicó por la mayor saliencia de las señales visuales en dicha condición. Estos resultados sugieren que los adultos mayores podrían requerir señales visuales más destacadas para interpretar con precisión las acciones de los demás por lo que el declive que experimentan en la sensibilidad a señales sutiles visuales, conduce a una mayor dependencia del proceso de análisis visual de la escena observada y su contexto semántico. $\mathrm{Si}$ se proyectan estas ideas desde el procesamiento visual al procesamiento del lenguaje, sería esperable que los mayores necesitaran la saliencia (propioceptiva e interoceptiva) del esfuerzo para lograr una comprensión más rápida y precisa, ya que el bajo esfuerzo (sobre todo en semánticas mentales más que físicas) generaría un desajuste en la interpretación, debido al declive en la sensibilidad a señales de esfuerzo más sutiles.

El presente estudio tuvo el propósito de investigar el impacto que tienen los declives cerebrales asociados al envejecimiento normal en los procesos de simulación sensoriomotora de oraciones de acción que impliquen esfuerzo, verificando las diferencias entre jóvenes y adultos mayores para semánticas de acción física y mental en contextos lingüísticos tanto factuales como contrafactuales. Nuestra predicción es que, al hallarse conservadas las funciones cognitivas relacionadas con el procesamiento semántico, incrementado el caudal léxico y dada la operatoria de mecanismos compensatorios (Salas-Herrera, 2015), los mayores experimentarán desbalances en la estimación del esfuerzo implicado en frases de semánticas abstractas, que luego podrían ser compensados. Para los mayores también se predicen desajustes más pronunciados en semánticas de acción física, ya que el deterioro físico en la vejez no experimenta mecanismos compensatorios de modo natural (la planificación, imaginería y ejecución motoras se ven alteradas en la vejez y tienen incidencia directa en los predictores). Al encontrarse alterados dichos componentes motores, se verá afectada la comprensión del lenguaje de acción (en comparación con el lenguaje mental y contrafactual).

Respecto a los jóvenes, no se esperan diferencias significativas, dado que su cuerpo y redes neurales relacionadas con la acción están indemnes. Esto quiere decir que no se esperan desbalances a nivel físico, sino más bien un 
efecto general del esfuerzo físico alto versus el bajo esfuerzo físico, es decir, que comprender frases de alto esfuerzo físico implique mayores niveles de imprecisión y/o mayor latencia. De existir desbalance es probable que ocurra a nivel de semánticas de verbos mentales, y en general, en frases con semánticas abstractas, debido a su menor experiencia y a que sus capacidades intelectuales aún no llegan a su plenitud (Hartshorne \& Germine, 2015). En todo caso, la tarea experimental es simple, por lo que no se espera una demanda excesiva y la probabilidad de desbalance es escasa.

\section{MÉTODO}

\section{Participantes}

Participaron 50 adultos mayores de la ciudad de Concepción $(M=66.18$ años, $\mathrm{DE}=4,39$, rango $=60-77,22$ mujeres y 28 hombres) y 43 jóvenes $(\mathrm{M}=21.28$ años, $\mathrm{DE}=1.08$, rango $=$ 19-24, 36 mujeres y 7 hombres) de la Universidad de Concepción. Se descartó cualquier tipo de problema cognitivo y de salud mental mediante el uso de pruebas cognitivas y psicopatológicas. Las pruebas aplicadas fueron Minimental State Examination (Folstein et al., 1983), Escala de Depresión Yesavage (Yesavage et al., 2000) en mayores y Escala de Depresión del Centro para Estudios Epidemiológicos en jóvenes (Gempp et al., 2004), más una breve encuesta construida por nuestro equipo que valoró funcionalidad (sobre todo en el plano sensoriomotor). Todos ellos con visión normal o corregida, diestros y hablantes nativos del español. Su participación fue gratuita y consentida, la que se registró mediante un protocolo validado por el Comité de Ética de la Universidad de Concepción. La duración media de esta evaluación fue de 15 minutos.

\section{Materiales y procedimientos}

Se elaboraron frases que correspondieran con ocho tipos de semánticas: alto esfuerzo físico real (AEF), bajo esfuerzo físico real (BEF), alto esfuerzo mental real (AEM), bajo esfuerzo mental real (BEM), alto esfuerzo físico en contrafactual (AECF), bajo esfuerzo físico en contrafactual (BECF), alto esfuerzo mental en contrafactual (AECM), bajo esfuerzo mental en contrafactual (BECM). Para tal efecto se construyeron 32 oraciones de cada una de las semánticas, que se distribuyeron en 4 bloques asignados aleatoriamente a cada participante. Además, cada sujeto leyó 20 oraciones sensorioperceptuales que no implicaban esfuerzo (EN) en modo indicativo y que sirvieron de relleno. En total, cada persona respondió a un bloque compuesto por 84 oraciones, las que se ejemplifican en la tabla I.

\section{Tabla 1}

Ejemplos de estímulos en la tarea de lectura

\begin{tabular}{|c|c|c|}
\hline İtem & Oraciones & Condición experimental \\
\hline $\mathrm{AEF}$ & Ahora/Estás/Enterrando /Un Cadáver/En La Fosa & Alto Esfuerzo Fisico Real \\
\hline $\mathrm{BEF}$ & Ahora/Estás/Enterrando/La Semilla/En El Huerto & Bajo Esfuerzo Físico Real \\
\hline AEM & Ahora/Estás/Resumiendo/La Materia/Para La Prueba & Alto Esfuerzo Mental Real \\
\hline BEM & Ahora/Estás/Resumiendo/Un Capitulo/De La Serie & Bajo Esfuerzo Mental Real \\
\hline $\mathrm{AECF}$ & Deberías/Haber/Atrapado/El Caballo/Con El Lazo & Alto Esfuerzo Físico Contrafactual \\
\hline BECF & Deberías/Haber/Atrapado/La Polilla/Con Tus Manos & Bajo Esfuerzo Físico Contrafactual \\
\hline $\mathrm{AECM}$ & Deberias/Haber/Compuesto/La Sinfonia/Para La Gala & Alto Esfuerzo Mental Contrafactual \\
\hline BECM & Deberías/Haber/Compuesto/La Rima/Para El Poema & Bajo Esfuerzo Mental Contrafactual \\
\hline EN & Ahora/Estás/Escuchando/La Canción/En El Concierto & Oración de relleno \\
\hline
\end{tabular}

Procedimientos y medidas de interés

Se usaron técnicas que permitiesen obtener medidas on-line durante la comprensión (Irrazábal \& Molinari, 2005): técnica de la ventana móvil (Just et al., 1982) y de reconocimiento rápido (Chang, 1980). En la primera, al comienzo de la tarea, los participantes observaron guiones y espacios en la pantalla del computador. Los guiones correspondían a las letras de cada palabra y los espacios a los lugares de segmentación de la oración. Al pulsar una tecla se desenmascaraba el segmento siguiente y se enmascaraba el que acababa de leer. En el reconocimiento rápido, luego de leer cada oración, se les presentó una palabra de prueba de modo que los sujetos debían decidir si dicha palabra estaba o no en la oración que acababan de leer presionando la letra P (la que se encontraba con una pegatina color rojo) para respuestas afirmativas y la letra Q (con pegatina 
verde) para respuestas negativas. Las medidas de interés registradas fueron el tiempo de lectura del objeto directo de cada frase y el tiempo de respuesta a la palabra de reconocimiento rápido. El experimento fue autoadministrado utilizando el software E-Prime 2.0 Professional.

\section{Diseño}

Las condiciones experimentales fueron analizadas en un diseño factorial de 2 Esfuerzo (alto vs. bajo) x 2 Imaginabilidad (alta vs. baja) x 2 Contexto (factual vs. contrafactual). Es importante aclarar que alta imaginabilidad correspondió a frases de verbos de acción física y baja imaginabilidad a frases usando verbos mentales.

\section{Análisis de datos}

El análisis de los datos se llevó a cabo mediante el empleo de un modelo lineal mixto. Este tipo de modelos permite examinar datos anidados. Esto resulta relevante para el presente estudio, dado que la variable dependiente analizada (tiempo de respuesta) se midió en ocho ocasiones para cada participante. Una ventaja adicional de este tipo de modelo es que no requiere que cada participante tenga el mismo número de mediciones en la variable dependiente medida. Como predictores de nivel-1 (intra-sujeto) se incluyeron los tres factores experimentales manipulados (esfuerzoimaginabilidad-contexto), mientras que como predictores de nivel-2 (entre-sujetos) se incluyeron género y edad.

Los análisis descritos previamente se llevaron a cabo con el programa Stata 15.0.

\section{RESULTADOS}

Para la comprensión de los resultados es importante considerar que el tiempo 4 corresponde al tiempo de lectura del objeto directo; el tiempo 5 es el tiempo para leer el complemento circunstancial y el tiempo 6 es el tiempo de lectura de la palabra de activación. La palabra "mental" en los gráficos y tablas corresponde al factor Imaginabilidad y "contrafactual" a Contexto.

\section{Análisis multinivel tiempo 4 (raíz cuadrada) correspondiente al objeto directo}

Inicialmente, se procedió a estimar un modelo que incluía los tres predictores de nivel-1, así como sus tres interacciones de dos vías y la interacción de tres vías. Para evaluar la necesidad de retener la interacción de tres vías, se llevó a cabo la aplicación de un likelihood ratio test (LRT) entre este modelo y un segundo modelo que excluía dicho efecto. El resultado del LRT llevó a exclusión de la interacción de tres vías del modelo $($ Chi2 $=0.08, p=0.78)$. A continuación, se procedió a analizar la necesidad de mantener las tres interacciones de dos vías mediante un LRT. Al comparar los modelos que incluían y excluían dichas interacciones, se llegó a la decisión de eliminar las tres interacciones (Chi2 $=1.53, p=$ 0.68).

En un segundo momento, se procedió a incluir al modelo los dos predictores de nivel-2, género y edad, así como las tres interacciones entre cada predictor de nivel-1 y la variable edad. Un LRT entre este modelo y un modelo que excluía las tres interacciones llevó a la decisión de excluir dichas interacciones (Chi2 $=6.75, p$ $=0.08)$. A continuación, se procedió a evaluar la contribución de las variables género y edad. Un LRT entre los modelos que incluían y excluían dichos predictores de nivel-2 llevó a la decisión de no retener dichos efectos en el modelo (Chi2 $=0.16, p=0.92$ ).

Los resultados del modelo final que sólo incluye los efectos de los tres factores experimentales (predictores de nivel-1) se presentan en la tabla 2. 
José Luis Salas-Herrera, Mabel Urrutia Martínez, Roberto Melipillan Araneda, et al.

Tabla 2

Variable dependiente tiempo 4 (raíz cuadrada)

\begin{tabular}{lrrrr}
\hline \multirow{2}{*}{ Variable } & \multirow{2}{*}{$\boldsymbol{\beta}$} & \multirow{2}{*}{ Error } & \multicolumn{2}{c}{ 95\% IC } \\
\cline { 4 - 5 } & -0.135 & 0.133 & Inferior & Superior \\
\hline Esfuerzo & $0.309^{* *}$ & 0.133 & 0.048 & 0.126 \\
Mental & $-0.856^{\text {*** }}$ & 0.133 & -1.117 & -0.594 \\
Contrafactual & -0.594 & \\
Intercepto & $27.703^{* * *}$ & 0.342 & 27.032 & 28.375 \\
\hline
\end{tabular}

$$
\text { Nota. }{ }^{*} p<0.05 ; * * p<0.01,{ }^{* * *} p<0.001
$$

De acuerdo con la tabla 2, tenemos que la propiedad de Imaginabilidad del texto impacta en los tiempos de lectura elevándolos $(\beta=0.309$; $p<0.05)$, mientras que la característica de contrafactual (contexto) hace que los tiempos de lectura disminuyan, como lo indica su signo $(\beta=-0.856 ; p<0.001)$. Ambos son efectos principales.

Análisis multinivel tiempo 5 (raíz cuadrada) correspondiente al complemento circunstancial

Inicialmente, se procedió a estimar un modelo que incluía los tres predictores de nivel-1, así como sus tres interacciones de dos vías y la interacción de tres vías. Para evaluar la necesidad de retener la interacción de tres vías, se llevó a cabo la aplicación de un likelihood ratio test (LRT) entre este modelo y un segundo modelo que excluía dicho efecto. El resultado del LRT llevó a exclusión de la interacción de tres vías del modelo $($ Chi2 $=0.33, p=0.56)$. A continuación, se procedió a analizar la necesidad de mantener las tres interacciones de dos vías mediante un LRT. Al comparar los modelos que incluían y excluían dichas interacciones, se llegó a la decisión de excluir la interacción correspondiente a esfuerzocontrafactual (Chi2 $=2.71, \mathrm{p}=0.10)$.

En un segundo momento, se procedió a incluir al modelo los dos predictores de nivel-2, género y edad, así como las tres interacciones entre cada predictor de nivel-1 y la variable edad. Un LRT entre este modelo y un modelo que excluía las tres interacciones con edad llevó a excluir dichas interacciones $(\mathrm{Chi} 2=4.81, \mathrm{p}=$ 0.19). A continuación, se procedió a evaluar la contribución de las variables género y edad. Un LRT entre los modelos que incluían y excluían dichos predictores de nivel-2 llevó a no retener dichos efectos en el modelo $(\mathrm{Chi} 2=0.34, \mathrm{p}=$ 0.84).

Los resultados del modelo final que sólo incluye los efectos de los tres factores experimentales (predictores de nivel-1) se presentan en la tabla 3.

Tabla 3

Variable dependiente tiempo 5 (raíz cuadrada)

\begin{tabular}{lrrrr}
\hline \multirow{2}{*}{ Variable } & \multirow{2}{*}{$\boldsymbol{\beta}$} & \multirow{2}{*}{ Error } & \multicolumn{2}{c}{$\mathbf{9 5 \%}$ CI } \\
\cline { 4 - 6 } & $1.049^{* * *}$ & 0.189 & Inferior & Superior \\
\hline Esfuerzo & 0.149 & 0.229 & -0.379 & 1.418 \\
Mental & $-0.508^{* *}$ & 0.188 & -0.877 & 0.599 \\
Contrafactual & $-0.732^{* *}$ & 0.266 & -1.253 & -0.210 \\
Mental-Esfuerzo & $0.611^{*}$ & 0.266 & 0.089 & 1.132 \\
Mental-Contrafactual & $26.944^{* * *}$ & 0.328 & 26.301 & 27.586 \\
Intercepto & &
\end{tabular}

Nota. ${ }^{p} p<0.05 ; * * p<0.01, * * p<0.001$

La tabla 3 muestra efectos principales de las propiedades textuales Esfuerzo $(\beta=1.049$; $p<0.001)$ y Contexto $(\beta=-0.508 ; p<$ $0.01)$. Aparecen también efectos de interacción Imaginabilidad-Esfuerzo $(\beta=-0.732 ; p<0.01) \mathrm{e}$ Imaginabilidad-Contexto $(\beta=0.611 ; p<0.05)$. En las figuras 1 y 2 se observa su dirección. Allí se aprecia que, para ambos efectos de interacción, tanto esfuerzo como contrafactual, modulan los tiempos de lectura en la medida que baja el nivel de imaginabilidad. En dicha condición, a mayor esfuerzo mayor tiempo de lectura, y a mayor propiedad contrafactual menor tiempo de lectura.

Figura 1.

Tiempo de lectura del complemento circunstancial como función del efecto de interacción Imaginabilidad-Esfuerzo

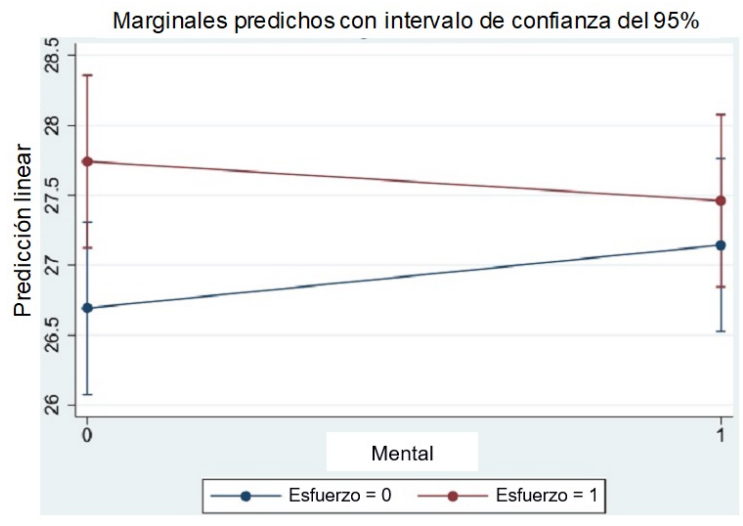


Figura 2.

Tiempo de lectura del complemento circunstancial como función del efecto de interacción

Imaginabilidad-Contexto

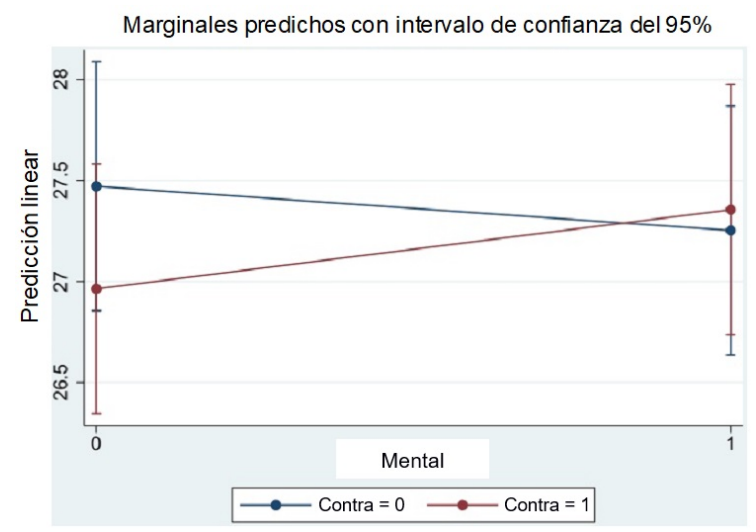

Análisis multinivel tiempo 6 (raíz cuadrada) correspondiente a la palabra de activación

Inicialmente, se procedió a estimar un modelo que incluía los tres predictores de nivel-1, así como sus tres interacciones de dos vías y la interacción de tres vías. Para evaluar la necesidad de retener la interacción de tres vías, se llevó a cabo la aplicación de un likelihood ratio test (LRT) entre este modelo y un segundo modelo que excluía dicho efecto. El resultado del LRT llevó a exclusión de la interacción de tres vías del modelo $($ Chi2 $=0.06, p=0.80)$. A continuación, se procedió a analizar la necesidad de mantener las tres interacciones de dos vías mediante un LRT. Al comparar los modelos que incluían y excluían dichas interacciones se excluyeron las interacciones correspondientes a imagineríaesfuerzo y esfuerzo-contexto (Chi2 $=2.99, p=$ 0.08).

En un segundo momento, se procedió a incluir al modelo los dos predictores de nivel-2, género y edad, así como las tres interacciones entre cada predictor de nivel-1 y la variable edad. Un LRT entre este modelo y un modelo que excluía las tres interacciones con edad, llevó a la decisión de excluir dichas interacciones $(\mathrm{Chi} 2=3.45, p=$ 0.33). A continuación, se procedió a evaluar la contribución de las variables género y edad. Un LRT entre los modelos que incluían y excluían dichos predictores de nivel-2 llevó a no retener dichos efectos en el modelo (Chi2 $=0.38, p=$ 0.83).

Los resultados del modelo final que sólo incluye los efectos de los tres factores experimentales (predictores de nivel-1) se presentan en la tabla 4.

\section{Tabla 4}

Variable dependiente tiempo 6 (raíz cuadrada)

\begin{tabular}{lrrrr}
\hline \multirow{2}{*}{ Variable } & \multirow{2}{*}{$\boldsymbol{\beta}$} & \multirow{2}{*}{ Error } & \multicolumn{2}{c}{$\mathbf{9 5 \%}$ CI } \\
\cline { 4 - 6 } & 0.049 & 0.170 & Inferior & \multicolumn{1}{c}{ Superior } \\
\hline Esfuerzo & -0.015 & 0.239 & -0.484 & 0.382 \\
Mental & $-0.986^{* * *}$ & 0.240 & -4.455 & -0.516 \\
Contrafactual & $0.727^{*}$ & 0.339 & 0.062 & 1.391 \\
Mental-Contrafactual & $30.220^{* * *}$ & 0.443 & 29.351 & 31.088 \\
Intercepto
\end{tabular}

Nota. ${ }^{*} p<0.05 ; * * p<0.01, * * * p<0.001$

La tabla 4 muestra el efecto principal de la propiedad textual contrafactual $(\beta=-0.986$; $p<0.001)$. Aparece también un efecto de interacción imaginabilidad-contexto $(\beta=0.727$; $p<0.05)$. En la figura 3 se observa su dirección. Allí se aprecia que la presencia del contrafactual disminuye los tiempos de lectura de la palabra de activación en la medida que baja la imaginabilidad de la frase.

\section{Figura 3.}

Tiempo de lectura de la palabra de activación como función del efecto de interacción mentalcontrafactual

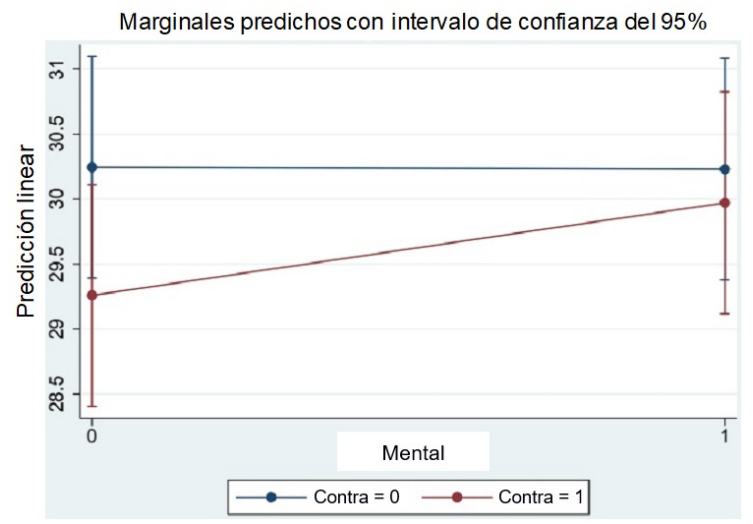

Cabe destacar que el análisis de los residuos de todos los modelos no mostró ningún incumplimiento de supuestos. 


\section{Discusión}

Los resultados indican que las propiedades de Esfuerzo, Imaginabilidad y Contexto son procesadas de manera similar en jóvenes y adultos mayores al momento de leer una frase, a lo largo de sus constituyentes sintagmáticos, así como al responder a una tarea de reconocimiento. Estos resultados forman parte de efectos principales que ejercen influencia en el procesamiento del objeto directo.

Posteriormente, el procesamiento semántico comienza a complejizarse, apareciendo efectos de interacción entre las propiedades del texto. El denominador común de estos efectos es la imaginabilidad. La interacción de imaginabilidad con las otras propiedades del texto parece operar de la siguiente forma: para el complemento circunstancial, si la imaginabilidad es baja, dicho segmento será afectados por el nivel de Esfuerzo, aumentando el tiempo de lectura y por el Contexto, disminuyendo el tiempo de lectura en la medida que aparece la propiedad contrafactual.

Por un lado, dado que se ha demostrado que el alto nivel de esfuerzo físico contenido en las frases es un parámetro corpóreo que se asocia con la planificación de acciones dirigidas a objetos, y favorece la simulación real del significado (Urrutia et al., 2012), hipotetizamos que el alto "nivel de esfuerzo mental", resultado novedoso y original de nuestro estudio, es un efecto que podría estar operando en un sentido similar y con un circuito cerebral similar o relacionado. Por otro lado, como se ha demostrado en otras investigaciones, los contrafactuales activan información corpórea al momento de recrear o simular el significado, debido a su doble representación del significado que activan el contenido corpóreo como si fuera real en un corto tiempo (de Vega \& Urrutia, 2011; de Vega \& Urrutia, 2012; Urrutia et al., 2012).

Ahora bien, el efecto de interacción imaginabilidad-contexto también incide en el procesamiento de la palabra de activación. Esto implica que, si el texto es de alta imaginabilidad, las propiedades del texto se procesan de forma similar y el efecto interactivo tenderá a desaparecer, evidenciando así el papel mediador de la condición de "baja imaginabilidad" en relación con esfuerzo y contexto. La propiedad de imaginabilidad se relaciona con qué tan bien una palabra da lugar a una imagen mental o experiencia sensorial. La palabra "auto", por ejemplo, suele tener una alta capacidad de imagen, mientras que la palabra "libertad" tienen una baja capacidad de imagen y esto se considera un índice psicolingüístico (p.e. Scott et al., 2019). De esta manera, y dado que la elicitación de imágenes o experiencias involucra un nivel de procesamiento sensorial y emocional mayor, entonces imaginería podría ser considerada una variable que refleja corporeidad y, a menor imaginabilidad, más abstracta y simbólica se vuelve la información que se procesa. Podemos entonces aseverar que nuestros datos dan cuenta de una versión de corporeidad que integra aspectos simbólicos con parámetros corpóreos, dado los efectos de interacción; aunque el factor predominante sigue siendo corpóreo, en este caso la imaginabilidad. Dicho tipo de corporeidad ha sido entendida teóricamente como corporeidad débil (Tirado et al., 2018), es decir, conjuntos de teorías que han encontrado respaldo empírico en la idea que las representaciones semánticas están constituidas, parcialmente, por información sensoriomotora (p.e. Mahon \& Hickok, 2016) con interacción del cuerpo y los procesos cognitivos (ver Tirado et al., 2018). Cualquier información sensorial o motora que se activa cuando tiene lugar el procesamiento semántico desempeña un rol de mediación en la información abstracta. En nuestro caso, Imaginabilidad medió en la expresión de los efectos Contexto y el Esfuerzo. Lo anterior permite afirmar que la comprensión del lenguaje en adultos mayores se encuentra conservada en cuanto al modo de procesar e integrar, transmodalmente, información abstracta y corpórea, lo que podría ser pensado como un índice de envejecimiento cognitivo exitoso.

El análisis de datos, por otro lado, evidenció que no aparece el efecto de la edad, lo que contradice los resultados de Bidet-Ildei et al. 
(2020). Es probable que esto se deba a las diferencias entre las características de ambas muestras, ya que nuestro estudio contó con la participación de 50 adultos mayores, $\mathrm{M}=$ 66.18 años, $\mathrm{DE}=4,39$, rango $=60-77,22$ mujeres y 28 hombres; y el de Bidet-Ildei et al. (2020) que contó con 20 participantes, $M=74.1$ años, $\mathrm{DE}=7.1$, rango $=65-84,17$ mujeres $\mathrm{y}$ 3 hombres, mostrando diferencias importantes en edad y género. El fundamento de nuestra decisión fue mantener coherencia con la edad de jubilación en nuestro país; y es probable que los adultos mayores de nuestra muestra aún se mantuvieran tan activos como aquellos en la adultez media, por lo que no se hallan menos corporeizados (Costello \& Bloesch, 2017). Esto abre proyecciones para comparar los hallazgos de nuestro estudio con otras cohortes etarias (de 75 y 85 años, por ejemplo), otros parámetros corpóreos, lenguas, usando palabras con mayor nivel de granularidad en cuanto a la experiencia sensorial y motora (Lynott et al., 2019) así como usando medidas neurocognitivas. Además, se destaca el potencial de intervención en adultos mayores con material lingüístico de carácter corpóreo como los parámetros de esfuerzo.

\section{CONCLUSIONES}

Nuestros datos avalan la validez evolutiva de la cognición corpórea (Vallet, 2015) en términos de que existen procesos corpóreos implicados en la constitución del significado lingüístico a lo largo de la vida, tanto en términos de efectos principales como de efectos interactivos. Los resultados apoyan una versión de corporeidad débil, dado que los aspectos simbólicos y corpóreos interactúan en la construcción del significado de cada frase de un modo transmodal y holístico con preponderancia de factores corpóreos como la imaginabilidad. "Baja imaginabilidad" parece ser la condición que media entre las propiedades Esfuerzo y Contexto. Esto significa que si, en el curso del procesamiento semántico, una zona o constituyente sintagmático se vuelve más abstracto, será más fácil procesarla si requiere de menor esfuerzo (físico o mental) y si ocurre en un contexto contrafactual, es decir, donde la implicación del cuerpo en la simulación perceptual y motora es hipotética y no real, como podría ser en un contexto factual, propio del modo indicativo.

Sería importante continuar la indagación con otras cohortes etarias (de 75 y 85 años, por ejemplo), otros parámetros corpóreos, lenguas, usando palabras o estímulos con mayor nivel de granularidad en cuanto a la experiencia sensorial y motora (Lynott et al., 2019) así como usando medidas neurocognitivas.

\section{Referencias}

Barsalou, L. W. (1999). Perceptions of perceptual symbols. Behavioral and brain sciences, 22(4), 637-660. https://doi.org/10.1017/SO $140525 X 99532147$

Bidet-Ildei, C., Beauprez, S. A., \& Boucard, G. (2020). The link between language and action in aging. Archives of gerontology and geriatrics, 90, 104099. https://doi.org/10.10 16/j.archger.2020.104099

Borghi, A. M., Barca, L., Binkofski, F., Castelfranchi, C., Pezzulo, G., \& Tummolini, L. (2019). Words as social tools: Language, sociality, and inner grounding in abstract concepts. Physics of life reviews, 29, 120-153. https://doi.org/10.1016/j.plrev.20 18.12.001

Borghi, A. M., Binkofski, F., Castelfranchi, C., Cimatti, F., Scorolli, C., \& Tummolini, L. (2017). The challenge of abstract concepts. Psychological Bulletin, 143(3), 263. https://d oi.org/10.1037/bul0000089

Chang, F. R. (1980). Active memory processes in visual sentence comprehension: Clause effects and pronominal reference. Memory Gु Cognition, 8(1), 58-64. https://doi.org/10 $.3758 / \mathrm{BF} 03197552$

Costello, M. C., \& Bloesch, E. K. (2017). Are Older Adults Less Embodied? A Review of Age Effects through the Lens of Embodied Cognition. Frontiers in psychology, 8, 267. ht tps://doi.org/10.3389/fpsyg.2017.00267 
José Luis Salas-Herrera, Mabel Urrutia Martínez, Roberto Melipillan Araneda, et al.

de Scalzi, M., Rusted, J., \& Oakhill, J. (2015). Embodiment effects and language comprehension in Alzheimer's disease. Cognitive science, 39(5), 890-917. https://do i.org/10.1111/cogs. 12187

de Vega, M., \& Urrutia, M. (2011). Counterfactual sentences activate embodied meaning: An action-sentence compatibility effect study. Journal of Cognitive Psychology, 23(8), 962-973. https: //doi.org/10.1080/20445911.2011.590471

de Vega, M., \& Urrutia, M. (2012). Discourse updating after reading a counterfactual event. Psicologica: International Journal of Methodology and Experimental Psychology, 33(2), 157-173. https://files.eric.ed.gov/full text/EJ973375.pdf

Diersch, N., Cross, E. S., Stadler, W., SchützBosbach, S., \& Rieger, M. (2012). Representing others' actions: the role of expertise in the aging mind. Psychological research, 76(4), 525-541. https://doi.org/10. 1007/s00426-011-0404-x

Dijkstra, K., Yaxley, R. H., Madden, C. J., \& Zwaan, R. A. (2004). The role of age and perceptual symbols in language comprehension. Psychology and Aging, 19(2), 352-356. https://doi.org/10.1037/08 82-7974.19.2.352

Di Rienzo, F., Joassy, P., Kanthack, T., MacIntyre, T. E., Debarnot, U., Blache, Y., Hautier, C., Collet, C., \& Guillot, A. (2019). Effects of action observation and action observation combined with motor imagery on maximal isometric strength. Neuroscience, 418, 82-95. https://doi.org/10.1016/j.neuroscien ce.2019.08.025

Folstein, M. F., Robins, L. N., \& Helzer, J. E. (1983). The mini-mental state examination. Archives of general psychiatry, 40(7), 812-812. https://doi.org/10.1001/arc hpsyc. 1983.01790060110016

Gallese, V. \& Lakoff, G. (2005). The brain's concepts: The role of the sensory-motor system in conceptual knowledge. Cognitive neuropsychology, 22 (3-4), 455-479. https://d oi.org/10.1080/02643290442000310
Gempp, R., Avendaño, C. \& Muñoz, C. (2004). Normas y punto de corte para la Escala de Depresión del Centro para Estudios Epidemiológicos (CES-D) en población juvenil chilena. Terapia Psicológica, 22(2), 145-156. https://www.redalyc.org/pdf/785/ 78522205.pdf

Gibson, James. (1979). The Ecological Approach to Visual Perception. Houghton Mifflin.

Glenberg, A., \& Gallese, V. (2012). Action-based language: A theory of language acquisition, comprehension, and production. Cortex, 48, 905-922. https://doi.org/10.1016/j.corte x.2011.04.010

Glenberg, A., \& Robertson, D. (2000). Symbol grounding and meaning: A comparison of high-dimensional and embodied theories of meaning. Journal of memory and language, 43(3), 379-401. https://doi.org/10.1006/jml a.2000.2714

Glenberg, A. M., Witt, J. K., \& Metcalfe, J. (2013). From the revolution to embodiment: 25 years of cognitive psychology. Perspectives on Psychological Science, 8(5), 573-585. https://doi.org/10.1 177/1745691613498098

Hooker, C., Roese, N. J., \& Park, S. (2000). Impoverished counterfactual thinking is associated with schizophrenia. Psychiatry, 63(4), 326-335. https://doi.org/10.1080/00 332747.2000 .11024925

Goudarzian, M., Ghavi, S., Shariat, A., Shirvani, H., \& Rahimi, M. (2017). Effects of whole body vibration training and mental training on mobility, neuromuscular performance, and muscle strength in older men. Journal of exercise rehabilitation, 13(5), 573. https:// doi.org/10.12965/jer.1735024.512

Guajardo, N. R., \& Cartwright, K. B. (2016). The contribution of theory of mind, counterfactual reasoning, and executive function to pre-readers' language comprehension and later reading awareness and comprehension in elementary school. Journal of Experimental Child Psychology, 144, 27-45. https://doi.org/10.1016/j.jecp.2 015.11 .004 
Hartshorne, J. K., \& Germine, L. T. (2015). When does cognitive functioning peak? The asynchronous rise and fall of different cognitive abilities across the life span. Psychological Science, 26(4), 433-443. https: //doi.org/10.1177/0956797614567339

Irrazábal, N. \& Molinari, C. (2005). Técnicas experimentales en la investigación de la comprensión del lenguaje. Revista Latinoamericana de Psicología, 37(3), 581-594. https://www.redalyc.org/pdf/805/ 80537309.pdf

Jamrozik, A., McQuire, M., Cardillo, E. R., \& Chatterjee, A. (2016). Metaphor: Bridging embodiment to abstraction. Psychonomic bulletin E⿱ review, 23(4), 1080-1089. https:/ /doi.org/10.3758/s13423-015-0861-0

Johnson, M. (2018). The Embodiment of Language. En A. Newen, L. de Bruin, \& S. Gallagher (Eds.) The Oxford Handbook of 4E Cognition (pp. 623-639). Oxford University Press.

Just, M. A., Carpenter, P. A., \& Woolley, J. D. (1982). Paradigms and processes in reading comprehension. Journal of experimental psychology: General, 111(2), 228-238. https ://doi.org/10.1037/0096-3445.111.2.228

Kenville, R., Maudrich, T., Carius, D., \& Ragert, P. (2017). Hemodynamic response alterations in sensorimotor areas as a function of barbell load levels during squatting: an fNIRS study. Frontiers in human neuroscience, 11, 1-13. https://doi.or g/10.3389/fnhum.2017.00241

Kulakova, E., \& Nieuwland, M. S. (2016). Understanding Counterfactuality: A Review of Experimental Evidence for the Dual Meaning of Counterfactuals. Language and Linguistics Compass, 10(2), 49-65. https://doi.org/10.1111/lnc3.12175

Loeffler, J., Raab, M., \& Cañal-Bruland, R. (2016). A Lifespan Perspective on Embodied Cognition. Frontiers in Psychology, 7, 1-6. https://doi.org/10.3389/f psyg.2016.00845

Louwerse, M. M. (2018). Knowing the meaning of a word by the linguistic and perceptual company it keeps. Topics in Cognitive
Science, 10(3), 573-589. https://doi.org/10. 1111/tops.12349

Lynott, D., Connell, L., Brysbaert, M., Brand, J., \& Carney, J. (2019). The Lancaster Sensorimotor Norms: multidimensional measures of perceptual and action strength for 40,000 English words. Behavior Research Methods, 1-21. https://doi.org/10.3758/s134 28-019-01316-z

Maguinness, C., Setti, A., Roudaia, E., \& Kenny, R. A. (2013). Does that look heavy to you? Perceived weight judgment in lifting actions in younger and older adults. Frontiers in Human Neuroscience, 7, 1-11. https://doi.or g/10.3389/fnhum.2013.00795

Mahon, B. Z., \& Hickok, G. (2016). Arguments about the nature of concepts: Symbols, embodiment, and beyond. Psychonomic bulletin Eु review, 23(4), 941-958. https://do i.org/10.3758/s13423-016-1045-2

Marmolejo-Ramos, F., Khatin-Zadeh, O., Yazdani-Fazlabadi, B., Tirado, C., \& Sagi, E. (2017). Embodied concept mapping: blending structure-mapping and embodiment theories. Pragmatics $\mathbb{E}$ Cognition, 24(2), 164-185. https://doi.org/1 0.1075/pc.17013.mar

Mohan, S., Mininger, A., \& Laird, J. (2016). Towards an Indexical Model of Situated Language Comprehension for Cognitive Agents in Physical Worlds. ar Xiv preprint arXiv: 1604.02509.

Nyhout, A., Henke, L., \& Ganea, P. A. (2019). Children's counterfactual reasoning about causally overdetermined events. Child development, 90 (2), 610-622. https://doi.org $/ 10.1111 /$ cdev. 12913

Ostarek, M., \& Huettig, F. (2019). Six challenges for embodiment research. Current Directions in Psychological Science, 28(6), 593-599. https://doi.org/10.1177/09 63721419866441

Paivio, A. (1986). Mental representations: A dual coding approach. Oxford University Press.

Ponari, M., Norbury, C. F., \& Vigliocco, G. (2018). Acquisition of abstract concepts is influenced by emotional valence. 
José Luis Salas-Herrera, Mabel Urrutia Martínez, Roberto Melipillan Araneda, et al.

Developmental science, 21(2), e12549. https ://doi.org/10.1111/desc.12549

Roese, N. J., \& Epstude, K. (2017). The functional theory of counterfactual thinking: New evidence, new challenges, new insights. Advances in experimental social psychology, 56, 1-79. https://doi.org/10.1016 /bs.aesp.2017.02.001

Romoli, J., Santorio, P., \& Wittenberg, E. (en prensa). Alternatives in Counterfactuals: What is right and what is not [No publicado]. https://semanticsarchive.net/A rchive $/ \mathrm{mEwMmQzN} /$ counterfactuals.pdf

Sadoski, M. (2018). Reading comprehension is embodied: Theoretical and practical considerations. Educational Psychology Review, 30(2), 331-349. https://doi.org/10.1 007/s10648-017-9412-8

Salas-Herrera, J. L. (2015). Lenguaje y envejecimiento desde una perspectiva corpórea. Paideia, (57), 43-64. https://revistasacademicas.udec.cl/i ndex.php/paideia/article/view/1495

Scorolli, C., Borghi, A. M., \& Glenberg, A. (2009). Language-induced motor activity in bi-manual object lifting. Experimental Brain Research, 193(1), 43-53. https://doi.o $\mathrm{rg} / 10.1007 / \mathrm{s} 00221-008-1593-4$

Scott, G. G., Keitel, A., Becirspahic, M., Yao, B., \& Sereno, S. C. (2019). The Glasgow Norms: Ratings of 5,500 words on nine scales. Behavior research methods, 51(3), 1258-1270. https://doi.org/10.3758/s13428 $-018-1099-3$

Springer, A., Huttenlocher, A., \& Prinz, W. (2012). Language-induced modulation during the prediction of others' actions. Psychological research, 76(4), 456-466. https ://doi.org/10.1007/s00426-012-0411-6

Ströbel, L. (2016). Sensory-motor concepts in language $\mathbb{E}$ cognition. University Press.

Tirado, C., Khatin-Zadeh, O., Gastelum, M., Leigh-Jones, N., \& MarmolejoRamos, F. (2018). The strength of weak embodiment. International Journal of Psychological Research, 11(2), 77-85. https:/ /doi.org/10.21500/20112084.3420
Ulrich, R., Eikmeier, V., de la Vega, I., Fernández, S. R., Alex-Ruf, S., \& Maienborn, C. (2012). With the past behind and the future ahead: Back-to-front representation of past and future sentences. Memory $\mathcal{E}$ cognition, 40(3), 483-495. https://doi.org/10.3758/s13 421-011-0162-4

Urrutia, M., Gennari, S. P., \& de Vega, M. (2012). Counterfactuals in action: an fMRI study of counterfactual sentences describing physical effort. Neuropsychologia, 50(14), 3663-3672. https://doi.org/10.1016 /j.neuropsychologia.2012.09.004

Vallet, G. T. (2015). Embodied cognition of aging. Frontiers in psychology, 6, 1-6. https://doi.or g/10.3389/fpsyg.2015.00463

Wolpert, D. M., Doya, K., \& Kawato, M. (2003). A unifying computational framework for motor control and social interaction. Philosophical Transactions of the Royal Society B: Biological Sciences, 358(1431), 593-602. https://doi.org/10.1098/rstb.2002.1238

Vukovic, N., Feurra, M., Shpektor, A., Myachykov, A., \& Shtyrov, Y. (2017). Primary motor cortex functionally contributes to language comprehension: An online rTMS study. Neuropsychologia, 96, 222-229. https:/doi.org/10.1016/ j.neuropsychologia.2017.01.025

Yang, Y., Dickey, M. W., Fiez, J., Murphy, B., Mitchell, T., Collinger, J., TylerKabara, E., Boninger, M. \& Wang, W. (2017). Sensorimotor experience and verbcategory mapping in human sensory, motor and parietal neurons. Cortex: a Journal Devoted to the Study of the Nervous System and Behavior, 92, 304-319. https://doi.org/1 0.1016/j.cortex.2017.04.021

Yesavage, J., Brink, T., Rose, T., \& Rush, A. (2000). Handbook of psychiatric measures: American Psychiatric Association.

Zwaan, R. A. (2014). Embodiment and language comprehension: reframing the discussion. Trends in cognitive sciences, 18(5), 229-234. https://doi.org/10.1016/j.tics.2014.02.008

\section{Notas}

* Artículo de investigación. 